\title{
Clinical and bacteriological survey of diabetic foot infections in Lisbon
}

\author{
J.J. Mendes ${ }^{a, *}$, A. Marques-Costa ${ }^{b}$, C. Vilela $^{c}$, J. Neves $^{d}$, N. Candeias ${ }^{e}$, \\ P. Cavaco-Silva ${ }^{f}$, J. Melo-Cristino $^{g}$ \\ a Internal Medicine Department, Hospital de Santa Marta/Centro Hospitalar de Lisboa Central EPE, Rua de Santa Marta, 50, 1169-024 Lisbon, \\ Portugal \\ ${ }^{\mathrm{b}}$ Podiatry Outpatient Clinic, Associação Protectora dos Diabéticos de Portugal, Lisbon, Portugal \\ ${ }^{\mathrm{c}}$ Immunology and Bacteriology Laboratory, Faculdade de Medicina Veterinária da Universidade Técnica de Lisboa, Lisbon, Portugal \\ d Surgery Department, Hospital de Santo António dos Capuchos/Centro Hospitalar de Lisboa Central EPE, Lisbon, Portugal

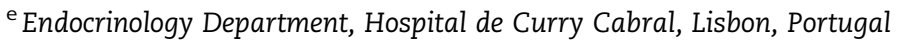 \\ ${ }^{\mathrm{f}}$ Instituto Superior de Ciências da Saúde - Egas Moniz, Caparica, Portugal \\ ${ }^{\mathrm{g}}$ Institute of Microbiology, Faculdade de Medicina de Lisboa, Lisbon, Portugal
}

\section{A R T I C L E I N F O}

\section{Article history:}

Received 31 July 2011

Received in revised form

16 September 2011

Accepted 3 October 2011

Published on line 21 October 2011

Keywords:

Epidemiology

Diabetic foot

Infection

Microbiology

Portugal

\begin{abstract}
A B S T R A C T
Aims: An epidemiological survey of diabetic foot infections (DFIs) in Lisbon, stratifying the bacterial profile based on patient demographical data, diabetic foot characteristics (PEDIS classification), ulcer duration and antibiotic therapy.

Methods: A transversal observational multicenter study, with clinical data collection using a structured questionnaire and microbiological products (aspirates, biopsies or swabs collected using the Levine method) of clinically infected foot ulcers of patients with diabetes mellitus (DM).

Results: Forty-nine hospitalized and ambulatory patients were enrolled in this study, and 147 microbial isolates were cultured. Staphylococcus was the main genus identified, and methicillin-resistant Staphylococcus aureus (MRSA) was present in $24.5 \%$ of total cases. In the clinical samples collected from patients undergoing antibiotic therapy, $93 \%$ of the antibiotic regimens were considered inadequate based on the antibiotic susceptibility test results. The average duration of an ulcer with any isolated multi-drug resistant (MDR) organism was 29 days, and previous treatment with fluoroquinolones was statistically associated with multidrug resistance.

Conclusions: Staphylococcus aureus was the most common cause of DFIs in our area. Prevalence and precocity of MDR organisms, namely MRSA, were high and were probably related to previous indiscriminate antibiotic use. Clinicians should avoid fluoroquinolones and more frequently consider the use of empirical anti-MRSA therapy.
\end{abstract}

(C) 2011 Elsevier Ireland Ltd. All rights reserved.

\footnotetext{
* Corresponding author. Tel.: +351 934855 822; fax: +351 213560368.

E-mail address: joaojoaomendes@hotmail.com (J.J. Mendes).

Abbreviations: CRTB, clinically relevant tissue burden; DFI, diabetic foot infection; DFU, diabetic foot ulcer; DM, diabetes mellitus; ESBL, extended-spectrum $\beta$-lactamase; HCP, health care provider; MDR, multi-drug resistant; MRSA, methicillin-resistant Staphylococcus aureus; PDR, pan-drug resistant.

0168-8227/\$ - see front matter (C) 2011 Elsevier Ireland Ltd. All rights reserved.

doi:10.1016/j.diabres.2011.10.001
} 


\section{Introduction}

Diabetes mellitus (DM) is a serious health problem that is rapidly expanding worldwide [1]. One of the more frequent diabetic complications is diabetic foot, which results from a complex interaction between a number of risk factors. Neuropathy (with alterations in motor, sensitive and autonomic functions) has a central role, causing ulcerations because of trauma or excessive pressure on deformed feet that lack protective sensitivity [2]. Once the protective layer of skin is broken, the deep tissues are exposed to bacterial colonization. Infections are facilitated by immunological deficits (especially in neutrophils), which are related to DM, and they rapidly progress to the deep tissues. Patients with DM frequently require minor or major amputations of the lower limbs (15-27\%), and in more than $50 \%$ of cases, infection is the preponderant factor [2].

Staphylococcus aureus is the most prevalent isolate in diabetic foot ulcers (DFUs), together with other aerobes (including Staphylococcus epidermidis, Streptococcus spp., Pseudomonas aeruginosa, Enterococcus spp. and coliform bacteria) and anaerobes [3,4]. Because of the polymicrobial nature of diabetic foot infections (DFIs), Karchmer and Gibbons [5] questioned the need for precisely defining the causative microorganism and suggested a treatment strategy based only on the knowledge of the general epidemiology. More recently, an increase in the incidence of multi-drug resistant (MDR) organisms, namely methicillin-resistant S. aureus (MRSA) and extended-spectrum $\beta$-lactamase (ESBL)-producing gram-negative bacteria, is threatening the outcome of anti-infectious therapy in the community and in hospitalized patients [4]. Therefore, the current guidelines [6] and expert opinion [7] advise providers to obtain specimens for culture before initiating empiric antibiotic therapy to help with the selection of a definitive therapy.

Although Portugal has one of the highest prevalences of DM, lower extremity amputations [8] and MRSA skin and soft tissue infections [9] in Europe, there is virtually no data on the prevalence and characterization of DFIs. Therefore, we performed an epidemiological survey of DFIs in Lisbon, stratifying the bacterial profiles based on patient demographical data, characteristics of diabetic foot (PEDIS classification), ulcer duration and current and recent ( $\leq 3$ months prior) antibiotic therapy.

\section{Subjects, materials and methods}

This transversal observational study was conducted at 4 clinical centers (2 outpatient clinics, 1 general surgery ward and 1 vascular surgery ward) in Lisbon from January 2010 to June 2010. A structured questionnaire was developed to record medical histories, examination details and investigation reports by health care providers (HCPs). Specimens were collected from patients with DM and clinically infected foot ulcers, as advised by current clinical guidelines [6]. A DFU was defined as a full-thickness wound below the ankle in a diabetic patient, irrespective of duration [10]. Infection was defined clinically by symptoms and signs of inflammation, as described by the infection item on the PEDIS system [10]. Specimens were obtained from patients before the first dose of antibiotics or while under antibiotic therapy with progression of infection signs and clinical deterioration of the ulcer.

This study was approved by the Faculty of Medicine of the University of Lisbon Research Ethics Committee and the Portuguese Data Protection Authority, and written informed consent was obtained for every patient.

\subsection{Clinical characterization}

For clinical characterization, 9 study factors were recorded for each patient: age, gender, DM duration (from diagnosis), last HbA1c value (accepted if collected in the last 3 months), hypertension and dyslipidemia (as defined according to the American Diabetes Association (ADA) guidelines for the diabetic population [11]), active tobacco abuse (defined as $\geq 20$ packs in the previous year), presence of ischemic heart disease (defined as previous history of myocardial infarction, coronary artery bypass graft or percutaneous transluminal coronary angioplasty) and chronic renal failure (defined as calculated glomerular filtration rate $<30 \mathrm{~mL} \mathrm{~min}^{-1} 1.73 \mathrm{~m}^{-2}$, permanent renal replacement therapy or previous transplant).

\subsection{Diabetic foot characterizations}

For characterization of diabetic foot, we used the International Working Group of the Diabetic Foot PEDIS system [10], which classified all foot ulcers in subcategories of five main categories (perfusion, extent/size, depth/tissue loss, infection and sensation), according to strict criteria. For the definition of osteomyelitis, a minimum of a positive probe-to-bone test [12] was accepted, but clinicians were encouraged to substantiate their diagnosis with the appropriate imaging studies. The number of previous ulcers and previous minor (toe or part of the foot) or major (above the ankle) amputations was also recorded.

\subsection{Antibiotic therapy}

HCPs were asked to register all current and recent (over the previous 3 months) antibiotic therapies.

\subsection{Collection of samples}

All HCPs were instructed on the proper methods for the collection of culture material, and a written protocol was provided. In the case of abscess with intact integument (and other closed lesions), the protocol suggested sampling by needle aspiration under strict aseptic technique. For ulcers and other open wounds, biopsy specimens were required, except in situations where the HCP considered that the invasive procedure could place the patient at risk (pain induction or risk of enlarging the ulcer). In only these situations, superficial swab samples were accepted, in strict accordance with the National Institute for Health and Clinical Excellence diabetic foot guideline [6]. For either of the procedures, debridement of necrotic tissue and cleansing with simple saline before sampling was obligatory. For 
biopsies, shaving or punch techniques, as previously described [13], were required. For swab sampling, HCPs were instructed on a standardized procedure [14], based on the Levine $1 \mathrm{~cm}^{2}$ swab method, using a flocked swab (ESwab Collection System, Copan).

\subsection{Transport}

Aspirates were transported in buffered isotonic agar with reduction agent media (Port-A-Cul Vial, BD BBL), and biopsies and swabs were transported in modified liquid Amies medium (ESwab Preservation System, Copan). Transport to the laboratory (Microbiology Laboratory, Faculty of Veterinary Medicine, Technical University of Lisbon) within $2 \mathrm{~h}$ of collection was assured by an on-call express courier.

\subsection{Processing and microbiological analysis of wound specimens}

Standard methods for sample processing and isolation and identification of aerobic and anaerobic bacteria were used [15]. Biopsy samples were weighed to the nearest milligram in sterile Petri dishes and homogenized in PBS using a pearl jar. A $100-\mu \mathrm{L}$ volume of the homogenate was used for serial dilutions in PBS. For aspirate samples, a $100-\mu \mathrm{L}$ volume of the recovered fluid was directly used for serial dilutions in PBS. Swab samples were vortexed with the swab inside for $5 \mathrm{~s}$, and then a $100-\mu \mathrm{L}$ volume of the suspension was used for serial dilutions in PBS. Quantification was performed using the 10-fold serial dilution method [15], and $100 \mu \mathrm{L}$ of each dilution was inoculated onto MacConkey agar (Merck)/Columbia ANC agar with 5\% sheep blood (BioMérieux) and, in duplicate, in Schaedler agar with $5 \%$ sheep blood (BioMérieux). The first two plates were incubated under aerobic conditions at $35^{\circ} \mathrm{C}$ for $24-48 \mathrm{~h}$, and the two Schaedler plates were incubated under anaerobic conditions (Anaerocult A, Merck) for 48-96 h. Additionally, samples were inoculated in Brain Heart Infusion Broth (Difco, BHIB) to allow recovery of fastidious or lowconcentration organisms. Isolates were identified by standard methods [15]. In some instances, unusual strains were identified using partial 16S rRNA gene sequencing [16]. Antimicrobial susceptibility testing of the aerobic isolates was performed using the standard disc diffusion method, as recommended by the Clinical and Laboratory Standards Institute [17]. Quantitative results were expressed in CFU/ $\mathrm{mL}$ for needle aspiration samples, CFU/g for biopsy samples and $\mathrm{CFU} / \mathrm{cm}^{2}$ for swab samples. Consistent with the study by Bill et al. [18] and the results of a recent systematic review [19], a swab count of $>10^{5} \mathrm{CFU} / \mathrm{cm}^{2}$ was considered equivalent to a tissue count of $>10^{5} \mathrm{CFU} / \mathrm{g}$ or a needle aspiration sample of $>10^{5} \mathrm{CFU} / \mathrm{mL}$; all of these values are considered to represent a clinically relevant tissue burden (CRTB).

\subsection{Multidrug resistance profiles}

Methicillin-resistant S. aureus (MRSA), methicillin-resistant S. epidermidis (MRSE) and other coagulase-negative Staphylococcus spp. (MRCN) were defined as strains phenotypically resistant to cefoxitin (by the disc diffusion method). Vancomycinresistant Enterococcus spp. (VRE) were defined as strains that were phenotypically resistant to vancomycin. (ESBL)-producing gram-negative strains were phenotypically confirmed using the cephalosporin/clavulanate combination disc test [20]. Multi-drug resistant (MDR) P. aeruginosa and Acinetobacter baumannii strains were defined as those resistant to at least three of six antibiotics, including amikacin, gentamicin, ciprofloxacin, piperacillin, ceftazidime and imipenem. Pandrug resistant (PDR) P. aeruginosa and A. baumannii/calcoaceticus strains were defined as those sensitive only to colistin [21]. All of these strains (MRSA, MRCN, VRE, [ESBL]-producing gramnegative bacteria, and MDR and PDR P. aeruginosa and A. baumannii/calcoaceticus) were considered to be MDR organisms.

\subsection{Statistical analyses}

Qualitative variables were expressed as percentages, and quantitative variables are expressed as means \pm SD (standard deviation). Significance of the study variables was tested using Student's t-test, the Chi-square test or Fisher's exact test, where appropriate. A two-tailed $p$ value of $<0.05$ was considered to be statistically significant. Additionally, the ulcer duration (in days) was stratified by microbial isolate and visually summarized in a box plot, with the boxes representing the lower and upper quartiles, the vertical line the median, the bars the minimum and maximum data points, and the solid diamond symbol the mean.

\section{Results}

A total of 49 patients (mean age of $62.7 \pm 12.7$ years and a maleto-female ratio of 6.8) were admitted during the study period. Their clinical and diabetic foot characteristics, stratified in accordance with the sample collection method, are shown in Table 1. Among these patients, the mean duration of DM was $23.0 \pm 12.8$ years, $26.5 \%$ had $\mathrm{HbA} 1 \mathrm{c}$ levels $<58 \mathrm{mmol} / \mathrm{mol}$ $(<7.5 \%),>90 \%$ had hypertension and/or dyslipidemia, and $30.6 \%$ and $10.2 \%$ had ischemic heart disease and chronic renal failure, respectively. Two-thirds of the patients had undergone recent antibiotic therapy, and one-third was currently undergoing antibiotic therapy. The majority of the samples came from outpatients (65.3\%), and swabbing was the most commonly used method (63.3\%) for sample collection. However, $92.8 \%$ of hospitalized patients and all clinically suspected osteomyelitis patients had samples collected by an invasive technique. There were statistically significant differences in the isolation rates of microorganisms from deep tissue samples and superficial swabs, with fewer aerobes per sample, in particular gram-positive bacteria ( $2.3 \pm 1.0$ vs. $1.3 \pm 1.2$ ), isolated from swabs, but there was no difference in the isolation rate of anaerobes or MDR organisms.

Out of the 49 patients enrolled in this study, 147 microbial isolates (comprising 43 species) were cultured, which represents an average of $3.0 \pm 1.4$ organisms per sample. Systematic results are presented in Table 2 . Aerobes were present in $98.0 \%$ of cases, with gram-positive bacteria comprising $66.0 \%$ of the total number of isolates. Staphylococcus was the main genus identified, with $S$. aureus present in $51 \%$ of the samples and in $94.1 \%$ of the cases with a CRTB. Coagulase-negative Staphylococcus spp. were the second most frequently encoun- 
Table 1 - Clinical and microbiological characteristics of DFIs stratified by the sample collection method.

\begin{tabular}{|c|c|c|c|}
\hline & Total $(n=49)$ & Swab samples $(n=31)$ & Deep tissue samples ${ }^{a}(n=18)$ \\
\hline Hospitalization (\%) & 34.7 & 12.9 & 72.2 \\
\hline \multicolumn{4}{|l|}{ Demographical data } \\
\hline Age (years) & $62.7 \pm 12.7$ & $60.2 \pm 13.5$ & $67.0 \pm 10.1$ \\
\hline Male gender (\%) & 83.7 & 87.1 & 77.8 \\
\hline \multicolumn{4}{|l|}{ Diabetes mellitus } \\
\hline Control of diabetes (HbA1c $<7 \%)$ & $20.4 \%$ & $16.1 \%$ & $17.8 \%$ \\
\hline Duration (years) & $23.0 \pm 12.8$ & $22.5 \pm 12.8$ & $23.7 \pm 13.1$ \\
\hline \multicolumn{4}{|l|}{ Co-morbidities } \\
\hline Hypertension (\%) & 93.9 & 96.8 & 88.9 \\
\hline Dyslipidemia (\%) & 95.9 & 93.4 & 100 \\
\hline Active tobacco abuse (\%) & 38.7 & 32.2 & 50.0 \\
\hline \multicolumn{4}{|l|}{ Organ lesions } \\
\hline Ischemic heart disease (\%) & 30.6 & 35.5 & 22.2 \\
\hline Chronic renal failure (\%) & 10.2 & 12.9 & 5.6 \\
\hline \multicolumn{4}{|l|}{ Diabetic foot characterization } \\
\hline Number of previous ulcers & $1.6 \pm 1.5$ & $1.9 \pm 1.6$ & $1.2 \pm 1.2$ \\
\hline Previous amputation (\%) & 46.9 & 51.6 & 38.9 \\
\hline Major & 10.2 & 9.7 & 11.1 \\
\hline Minor & 38.8 & 45.2 & 27.8 \\
\hline Duration of present ulcer (days) & $30.6 \pm 31.9$ & $33.4 \pm 25.9$ & $25.7 \pm 40.5$ \\
\hline Neuroischemic (\%) & 53.1 & 54.8 & 50.0 \\
\hline Osteomyelitis (\%) & 30.6 & 0.0 & 83.3 \\
\hline \multicolumn{4}{|l|}{ PEDIS } \\
\hline \multicolumn{4}{|l|}{ Perfusion } \\
\hline $1(\%)$ & 44.9 & 43.9 & 46.6 \\
\hline $2(\%)$ & 40.8 & 40.7 & 41.0 \\
\hline $3(\%)$ & 14.3 & 19.3 & 12.4 \\
\hline Extent $\left(\mathrm{cm}^{2}\right)$ & $13.3 \pm 56.9$ & $1.2 \pm 0.6$ & $34.3 \pm 91.7$ \\
\hline \multicolumn{4}{|l|}{ Depth } \\
\hline $1(\%)$ & 18.4 & 29.0 & 0.1 \\
\hline $2(\%)$ & 51.0 & 71.0 & 16.6 \\
\hline $3(\%)$ & 30.6 & 0.0 & 83.3 \\
\hline \multicolumn{4}{|l|}{ Infection } \\
\hline $2(\%)$ & 61.2 & 87.1 & 16.6 \\
\hline $3(\%)$ & 36.7 & 12.9 & 77.7 \\
\hline $4(\%)$ & 2.0 & 0.0 & 5.4 \\
\hline \multicolumn{4}{|l|}{ Sensation } \\
\hline $2(\%)$ & 100 & 100 & 100 \\
\hline \multicolumn{4}{|l|}{ Antibiotic therapy } \\
\hline Previous (\%) & 65.3 & 67.7 & 61.2 \\
\hline Current (\%) & 30.6 & 23.0 & 43.7 \\
\hline \multicolumn{4}{|l|}{ Isolates } \\
\hline Monomicrobial (\%) & 16.3 & 12.9 & 22.1 \\
\hline Total number (per sample) & $3.0 \pm 1.4$ & $3.2 \pm 1.3$ & $2.7 \pm 1.4$ \\
\hline Aerobes & $2.5 \pm 1.1$ & $2.7 \pm 0.9$ & $2.3 \pm 1.3$ \\
\hline Gram-positive & $2.0 \pm 1.0$ & $2.3 \pm 1.0$ & $1.6 \pm 1.1$ \\
\hline Gram-negative & $0.6 \pm 0.6$ & $0.5 \pm 0.5$ & $0.7 \pm 0.7$ \\
\hline Anaerobes & $0.4 \pm 0.6$ & $0.4 \pm 0.6$ & $0.3 \pm 0.6$ \\
\hline MDR organisms & $0.6 \pm 0.9$ & $0.5 \pm 0.8$ & $0.9 \pm 1.0$ \\
\hline
\end{tabular}

${ }^{a}$ Biopsies $(n=14)$ and aspirates $(n=4)$.

MDR: multi-drug resistant.

tered aerobic gram-positive isolates, with S. epidermidis and Staphylococcus lugdunensis commonly associated with a CRTB. Corynebacterium spp. and other uncommon gram-positive bacteria were also identified but not in clinically significant quantities. Streptococcus spp. were infrequently (4.1\%) isolated. Gram-negative aerobes comprised $19.0 \%$ of the isolated organisms, while $P$. aeruginosa, the single most predominant species, was isolated in only $12.2 \%$ of cases. Proteus spp. were the next most frequently recovered gram-negative bacteria, although largely (75.0\%) in non-CRTB cases. A. baumannii/ calcoaceticus were identified in $8.2 \%$ of the cases and were the
non-PDR species found exclusively in the non-CRTB cases. Anaerobes were found in $30.6 \%$ of patients, with Peptostreptococcus spp. accounting for $55.0 \%$ of all anaerobic isolates, followed by the Bacteroides fragilis group, which accounted for $25 \%$ of these isolates, but this last group was more frequently identified in non-CRTB. Candida spp. were infrequently encountered, representing only $1.4 \%$ of the total isolates.

MDR organisms were present in $38.8 \%$ of cases, while MRSA was found in $24.5 \%$ of patients, thereby making it the predominantly isolated pathogen. MRSE and other methicillin-resistant coagulase-negative Staphylococci were also iden- 
Table 2 - Distribution of the DFI isolates.

\begin{tabular}{|c|c|c|c|c|}
\hline & $n$ & $\%$ & $\%$ (/patients) & CRTB \\
\hline Aerobes & 125 & 85.0 & 98.0 & $63.2 \%$ \\
\hline Gram-positive & 97 & 66.0 & 95.9 & $64.9 \%$ \\
\hline Staphylococcus spp. & 54 & 36.7 & 79.6 & $66.7 \%$ \\
\hline Staphylococcus aureus (MRSA) & $32(17)$ & $21.8(11.6 \%)$ & $51.0(24.5 \%)$ & $93.8 \%(94.1 \%)$ \\
\hline Staphylococcus epidermidis (MRSE) & $7(3)$ & $4.8(2.0 \%)$ & $14.3(4.1 \%)$ & $42.9 \%(66.7 \%)$ \\
\hline Other coagulase-negative Staphylococcus spp. $(\mathrm{MRCN})^{\mathrm{a}}$ & $15(3)$ & $10.2(2.0 \%)$ & $20.4(4.1 \%)$ & $20.0 \%(33.3 \%)$ \\
\hline Streptococcus spp. ${ }^{\mathrm{b}}$ & 6 & 4.1 & 12.2 & $100 \%$ \\
\hline Enterococcus spp. ${ }^{\mathrm{c}}$ (VRE) & $13(1)$ & $8.8(0.7 \%)$ & $20.4(2.0 \%)$ & $76.9 \%(100 \%)$ \\
\hline Corynebacterium spp. ${ }^{\mathrm{d}}$ & 12 & 8.2 & 28.6 & $50.0 \%$ \\
\hline Other Gram-positives ${ }^{e}$ & 12 & 8.2 & 22.4 & $41.7 \%$ \\
\hline Gram-negative & 28 & 19.0 & 51.0 & $57.1 \%$ \\
\hline Enterobacteriaceae & 16 & 10.9 & 16.3 & $56.3 \%$ \\
\hline Escherichia coli & 1 & 0.7 & 2.0 & $100 \%$ \\
\hline Klebsiella spp. (ESBL) & $2(1)$ & $1.4(0.7 \%)$ & $4.1(2.0 \%)$ & $100 \%(100 \%)$ \\
\hline Proteus spp. ${ }^{\mathrm{f}}$ & 8 & 5.4 & 16.3 & $25.0 \%$ \\
\hline Other Enterobacteriaceae ${ }^{g}$ & 5 & 3.4 & 4.1 & $80.0 \%$ \\
\hline Nonfermenting negative bacilli & 12 & 8.2 & 20.4 & $58.3 \%$ \\
\hline MDR Pseudomonas aeruginosa (PDR-PA) & $7(2)$ & $4.8(1.4 \%)$ & $12.2(4.1 \%)$ & $71.4 \%(100 \%)$ \\
\hline MDR Acinetobacter baumannii/calcoaceticus (PDR-AB) & $5(3)$ & $3.4(2.0 \%)$ & $8.2(6.1 \%)$ & $40.0 \%(66.7 \%)$ \\
\hline Anaerobes & 20 & 13.6 & 30.6 & $75.0 \%$ \\
\hline Peptostreptococcus spp. & 11 & 7.5 & 22.4 & $100 \%$ \\
\hline Bacteroides fragilis group & 5 & 3.4 & 4.1 & $20.0 \%$ \\
\hline Other anaerobes ${ }^{\mathrm{h}}$ & 4 & 2.7 & 4.1 & $75.0 \%$ \\
\hline Yeasts $^{\mathrm{i}}$ & 2 & 1.4 & 4.1 & - \\
\hline \multicolumn{5}{|c|}{$\begin{array}{l}\text { In brackets are the multi-drug resistant (MDR) organisms of each species; CRTB: clinically relevant tissue burden; MRSA: methicillin-resistant } \\
\text { Staphylococcus aureus; MRSE: methicillin-resistant Staphylococcus epidermidis; MRCN: methicillin-resistant coagulase-negative Staphylococcus spp. } \\
\text { other than Staphylococcus epidermidis; VRE: vancomycin-resistant Enterococci; ESBL: extended-spectrum beta-lactamases producing Enterobacter- } \\
\text { iaceae; MDR: multi-drug resistant; PDR-PA/PDR-AB: pan-drug-resistant Acinetobacter baumannii/pandrug-resistant Pseudomonas aeruginosa. } \\
\text { a Staphylococcus lugdunensis }(n=2) \text { and other coagulase-negative Staphylococcus spp. }(n=13) \text {. } \\
\text { b Streptococcus agalactiae }(n=3) \text {, Streptococcus mitis group }(n=1) \text { and Streptococcus dysgalactiae }(n=2) \text {. } \\
\text { c Enterococcus faecalis }(n=9) \text { and Enterococcus faecium }(n=1) \text {. } \\
\text { d Corynebacterium amycolatum/striatum }(n=9) \text { and other Corynebacterium spp. }(n=3) \text {. } \\
\text { e Dermabacter hominis }(n=1) \text {, Leuconostoc spp. }(n=1) \text {, Arcanobacterium spp. }(n=2) \text {, Arthrobacter spp. (n=1), Kocuria varians/rosea ( }(n=2) \text {, } \\
\text { Cellulomonas spp./Micrococcus spp. ( } n=1) \text { and Brevibacterium spp. }(n=4) \text {. } \\
\text { f Proteus mirabilis }(n=4) \text { and Proteus vulgaris }(n=4) \text {. } \\
\text { g Enterobacter spp. }(n=1) \text {, Serratia marcescens }(n=2) \text { and Morganella morganii }(n=2) \text {. } \\
\text { h Fusobacterium spp. }(n=1) \text {, Prevotella spp. }(n=1) \text {, Eggerthella spp. }(n=1) \text { and Veinonella spp. }(n=1) \text {. }\end{array}$} \\
\hline
\end{tabular}

tified but accounted for only $4.8 \%$ of the isolates. Gramnegative MDR organisms were identified in a total of $18.9 \%$ of the patients. Of the isolated A. baumannii and P. aeruginosa strains, $38.5 \%$ were PDR, and the remainder were MDR.

Although a longitudinal study using sequential microbiological samples was not performed, visually representing the relationship between the microbial isolates and ulcer duration in a box plot graph (Fig. 1) revealed a pattern: gram-positive bacteria appeared in ulcers of short duration, while anaerobes associated with either gram-positive or -negative organisms appeared in ulcers of longer duration. This finding was independent of previous or current antimicrobial therapy. The average duration of an ulcer with any isolated MDR organism was 29 days.

In the clinical samples collected from patients undergoing antibiotic therapy (Table 3), which corresponded mainly to hospitalized patients with osteomyelitis, $93 \%$ of the antibiotic regimens were considered inadequate based on the antibiotic susceptibility test results. Quantitative and qualitative differences were found in these samples, with fewer microorganisms identified $(2.1 \pm 0.9$ vs. $3.4 \pm 1.3)$; in particular, fewer gram-positive ( $86.7 \%$ vs. $100 \%$ ) and anaerobic (6.7\% vs. $41.2 \%)$ bacteria were identified; however, there was a higher prevalence of MDR organisms (66.7\% vs. $26.5 \%$ ). Although all the clinical variables were examined, multi-drug resistance was only statistically associated with current antibiotic treatment (with any class of antibiotics) and with previous fluoroquinolone treatment (Table 4).

\section{Discussion}

DFIs are common, complex, and costly. They account for the largest number of proximate nontraumatic lower extremity amputations [2]. This public health problem is particularly important in the underdiagnosed and undertreated diabetic Portuguese population [8]. To our knowledge, this is the first published epidemiological study that reports the infectious microbiota and clinical characteristics of diabetic foot in patients located in Portugal. This study reflects the clinical profiles of inpatients and outpatients in the Lisbon area, but because the sample was relatively small, the study population was heterogeneous, and some controversial methodological issues were utilized (notably, the use of swabs and quantita- 


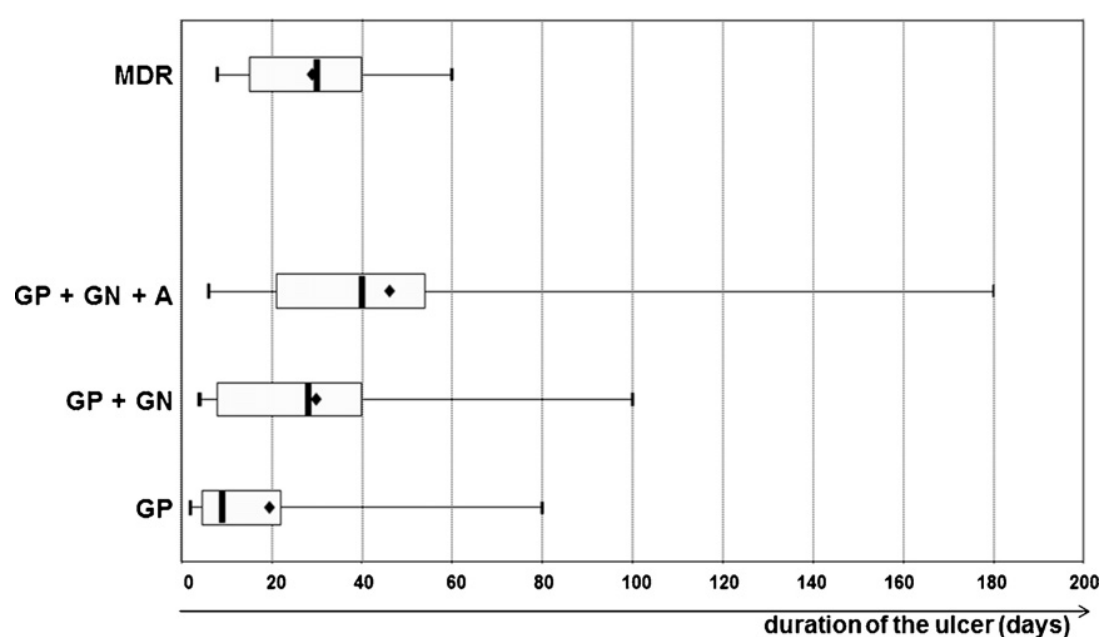

Fig. 1 - A box plot representing the ulcer duration data (in days), stratified by the microbial isolate (the boxes represent the lower and upper quartiles, the vertical line the median, the bars the minimum and maximum data points, and the solid diamond symbol the mean). MDROs: multi-drug resistant organisms, GP: gram-positive aerobes, GN: gram-negative aerobes, and A: anaerobes.

tive results), care must be taken when interpreting these results.

The baseline characteristics of the sample population are in line with those previously reported by European DFU studies [22], except for the high percentage of male patients and low percentage of patients with controlled DM (as evaluated by HbA1c). This can be partially explained by the hypothesis of a recent study [23] that reported that male gender and poor glycemic control are independent risk factors for infection and non-healing DFUs. The high prevalence of comorbidities is due to the low cut-offs used in the definitions.
Clinical guidelines [6] use infection severity and other clinical characteristics of DFUs as the basis for selecting an appropriate treatment approach, including antibiotic therapy. Our study used the PEDIS classification, and there were no statistical relationships between the diabetic foot characteristics, other than the duration of the ulcer and a clinical suspicion of osteomyelitis, and specific pathogens. We cannot be certain that the lack of significant associations was due only to the small sample size, however.

It is well documented in the literature $[3,4]$ that DFIs are polymicrobial in nature. In the present study, polymicrobial

Table 3 - Distribution of the DFI isolates in relation to current antibiotic therapy.

\begin{tabular}{|c|c|c|c|c|}
\hline & $\begin{array}{c}\text { Total } \\
(n=49)\end{array}$ & $\begin{array}{c}\text { Not under antibiotic } \\
\text { therapy }(n=34)\end{array}$ & $\begin{array}{l}\text { Under antibiotic } \\
\text { therapy }(n=15)\end{array}$ & $p^{\mathrm{a}}$ \\
\hline Hospitalization (\%) & 34.7 & 17.6 & 73.3 & $<0.01$ \\
\hline \multicolumn{5}{|l|}{ Isolates } \\
\hline Total number (per sample) & $3.0 \pm 1.4$ & $3.4 \pm 1.3$ & $2.1 \pm 0.9$ & $<0.01$ \\
\hline \multicolumn{5}{|l|}{ Aerobes } \\
\hline Number present per sample & $2.5 \pm 1.1$ & $2.9 \pm 1.0$ & $1.9 \pm 1.0$ & $<0.01$ \\
\hline Samples with $\geq 1(\%)$ & 98.0 & 100 & 93.3 & NS \\
\hline \multicolumn{5}{|l|}{ Gram-positive } \\
\hline Number present per sample & $2.0 \pm 1.0$ & $2.3 \pm 1.0$ & $1.5 \pm 1.1$ & 0.02 \\
\hline Samples with $\geq 1$ (\%) & 95.9 & 100 & 86.7 & 0.03 \\
\hline \multicolumn{5}{|l|}{ Gram-negative } \\
\hline Number present per sample & $0.6 \pm 0.6$ & $0.6 \pm 0.6$ & $0.4 \pm 0.6$ & NS \\
\hline Samples with $\geq 1$ (\%) & 51.0 & 58.8 & 33.3 & NS \\
\hline \multicolumn{5}{|l|}{ Anaerobes } \\
\hline Number present per sample & $0.4 \pm 0.6$ & $0.5 \pm 0.6$ & $0.1 \pm 0.5$ & NS \\
\hline Samples with $\geq 1$ (\%) & 30.6 & 41.2 & 6.7 & 0.01 \\
\hline \multicolumn{5}{|l|}{ MDR organisms } \\
\hline Number present per sample & $0.6 \pm 0.9$ & $0.4 \pm 0.7$ & $1.1 \pm 1.0$ & $<0.01$ \\
\hline Samples with $\geq 1(\%)$ & 38.8 & 26.5 & 66.7 & $<0.01$ \\
\hline Antibiotic therapy covers isolated pathogens & - & - & $7.0 \% \mathrm{~b}$ & - \\
\hline
\end{tabular}


Table 4 - Relationship between MDR organisms and recent ( $\leq 3$ months) or current antibiotic therapy.

\begin{tabular}{|c|c|c|c|}
\hline & Non-MDR $(n=30)$ & $\operatorname{MDR}^{a}(n=19)$ & $p^{b}$ \\
\hline Previous antibiotic therapy & $63.3 \%$ & $73.7 \%$ & NS \\
\hline Penicillins (including associations with $\beta$-lactamase inhibitors) & $63.3 \%$ & $79.0 \%$ & NS \\
\hline Cephalosporins & $13.3 \%$ & $26.3 \%$ & NS \\
\hline Carbapenems & $10.0 \%$ & $5.3 \%$ & NS \\
\hline Aminoglycosides & $0.0 \%$ & $0.0 \%$ & NS \\
\hline Sulphamides & $13.3 \%$ & $15.8 \%$ & NS \\
\hline Fluoroquinolones & $23.3 \%$ & $63.2 \%$ & $<0.01$ \\
\hline Glycopeptides & $6.7 \%$ & $5.3 \%$ & NS \\
\hline Oxazolidinones & $0.0 \%$ & $5.3 \%$ & NS \\
\hline Others & $3.3 \%$ & $5.3 \%$ & NS \\
\hline Current antibiotic therapy & $16.7 \%$ & $52.6 \%$ & $<0.01$ \\
\hline Penicillins (including associations with $\beta$-lactamase inhibitors) & $6.7 \%$ & $0.0 \%$ & NS \\
\hline Cephalosporins & $0.0 \%$ & $0.0 \%$ & NS \\
\hline Carbapenems & $10.0 \%$ & $15.8 \%$ & NS \\
\hline Aminoglycosides & $0.0 \%$ & $5.3 \%$ & NS \\
\hline Sulphamides & $3.3 \%$ & $0.0 \%$ & NS \\
\hline Fluoroquinolones & $10.0 \%$ & $15.8 \%$ & NS \\
\hline Glycopeptides & $3.3 \%$ & $5.3 \%$ & NS \\
\hline Oxazolidinones & $0.0 \%$ & $5.3 \%$ & NS \\
\hline Others & $0.0 \%$ & $5.3 \%$ & NS \\
\hline Covers the isolated pathogens & $40.0 \%$ & $0.0 \%$ & 0.03 \\
\hline
\end{tabular}

cultures were obtained from $83.7 \%$ of patients with a rate of isolation of $3.0 \pm 1.4$ bacteria per patient, independent of the sampling method, which is similar to the results seen in previous studies. In agreement with published western studies [3,4], we isolated predominantly aerobic gram-positive cocci from acute infections, while a more complex flora, including gram-negative and anaerobic bacteria was obtained from chronic wounds.

We also found that $S$. aureus, either alone or as a component of a mixed infection, to be the most frequently isolated pathogen. Coagulase-negative Staphylococcus spp. were also frequently found, often with a methicillin-resistance phenotype. Streptococcus spp., which are well-recognized pathogens in DFIs, were infrequently isolated. This can be partially justified by the high prevalence of present and recent antibiotic therapy. Enterococcus spp., considered low-virulence commensal organisms, except in diabetic and other compromised patients, were identified in $20.4 \%$ of patients, which is in accordance with other studies $[3,4]$.

In strict accordance with other western studies [3,4], but unlike studies from India and other Asian countries [24], we isolated relatively few aerobic gram-negative organisms.

In our study, the high percentage of $P$. aeruginosa and low percentage of Proteus spp. isolates with a CRTB was consistent with the view that the first species can cause severe tissue damage in DM patients and should be regarded as significant in that population, while the latter are most commonly nonpathogenic [7].

Independent of the sampling method, anaerobes were isolated in one-third of the patients and almost always in mixed culture. This is in contrast to the findings of several other studies that failed to isolate anaerobes, possibly because of suboptimal study protocols [25]. The anaerobes isolated from our study are consistent with other reported studies [26], in which Peptostreptococcus spp. were the predominant isolates.
Although the exact role of anaerobic bacteria in DFIs is still under debate, our study is in line with the expert opinion [7] that suggests that anaerobes are more likely to be isolated from long-standing infections.

Other important factors to consider when interpreting the results of our study are that DFI is a clinical diagnosis and that both the quantitative and qualitative aspects of wound microbiology are critical determinants of an infection's course. All the patients enrolled in our study had clinically infected DFUs, and we based our conclusions on a qualitative microbiological analysis, considering the diversity of the microorganisms and the potential for microbial synergy, and on quantitative microbiological analysis, which provided a good indication of the microbial load. Assuming that the qualitative microbiology remains constant, the probability of wound infection increases with the microbial load, up to a critical level at which infection or a failure to heal is considered to be almost inevitable. In this paper, CRTB represented the quantitative aspect of wound microbiology and was used only as a potential indicator of the microorganisms' relevance in clinically infected DFUs.

One of the main limitations of our study is that the quantitative and qualitative microbial evaluations were predominantly performed using swab samples. While tissue biopsies and fluid aspirates are considered the gold standard for diagnosing wound infections [25], these invasive tests are performed infrequently with small wounds and in many practice settings, such as outpatient clinics, due to concerns over enlarging the ulcer or inducing pain $[14,25,27]$. In our study, we introduced a standardized procedure that was strictly consistent with the current clinical guidelines [6]. Our method used quantitative aerobic and anaerobic swab cultures as an alternative method when the HCP believed an invasive procedure would place the patient at risk. While this decision was based on the microbiological experimental 
and clinical evidence supporting the hypothesis that the results form quantitative swabs are highly correlated with those from invasive procedures (sensitivities from $93.5 \%$ to $100 \%$ and specificities from $76.3 \%$ to $94.2 \%$ have been previously reported [14]), this hypothesis is not consensual in the scientific community. Some authors have reported consistency between swab and deep tissue biopsy sample cultures [28,29], while others believe that superficial swab cultures of DFIs only complicate patient evaluation by sampling the superficial wound compartment, which may contain colonizing organisms rather than true pathogens. These divergent conclusions may be explained by different and non-standardized protocols. While we acknowledge that a standardized quantitative swab sampling protocol may be an imperfect and difficult-to-implement method in the clinical setting, it clearly has merits in the research field, at least in a setting with a high prevalence of the multi-drug resistance setting such as in our study; when properly interpreted, they can provide useful information [27].

We had a surprisingly high number of swab samples (mainly from outpatient clinics) from patients with small superficial ulcers. There were statistically significant differences between the superficial and deep samples, probably due to swab-associated and impossible-to-eliminate wound contamination by members of the endogenous microbiota (mainly gram-positive aerobes). This result may explain the high prevalence of Corynebacterium spp. and other lowvirulence colonizers (e.g., Dermabacter hominis and Leuconostoc spp.), which were mainly cultured from swab samples.

In the present study, MDR organisms were cultured from $38.8 \%$ of the patients, the majority $(24.5 \%)$ of which were MRSA. Most of the other international studies that have reported a similarly high percentage of MDR organisms were single-center, hospital-based studies [24]. The high prevalence in such studies may be explained by the institution's use of broad spectrum antibiotics, resulting in a pathogen-selective survival advantage. In our multicenter study, we did not find any statistically significant differences between the inpatients and outpatients, and the mean duration of ulcers with isolated MDR organisms was short (29 days).

We also found a high percentage of patients (65.3\%) who had received antibiotics in the previous three months and a statistical association between the presence of MDR organisms and previous fluoroquinolone therapy. This class of antibiotics has been widely used in Portugal for many years [30], and others have described [31] how they use correlates with the spread of MDR organisms, particularly MRSA. Therefore, our results suggest that multi-resistance in our area is widespread in diabetic patients with foot ulcers, and fluoroquinolone abuse (including inadequate dosing or suboptimal therapy duration) in the community could be a potential cause.

We also evaluated samples from DFI patients receiving antibiotic therapy, mainly hospitalized patients with osteomyelitis, who had signs of infection progression and clinical deterioration of their ulcers. Microbial isolation was significantly influenced by systemic antibiotic therapy, with fewer microorganisms (mostly anaerobic bacteria) identified but with a significantly greater prevalence of MDR organisms. This finding may be explained by selective pressure because the majority of these patients were under broad-spectrum antibiotic therapy, mostly with carbapenems. There are surprisingly few published clinical trials on antibiotic therapy for DFIs, and the available data do not allow current guidelines to recommend any specific antibiotic regimen. In 2010, however, the Portuguese Directorate-General of Health [32] published a clinical guideline suggesting the use of isoxazolylpenicillins or clindamycin for superficial infections, aminopenicillins with a $\beta$-lactamase inhibitor or fluoroquinolones combined with clindamycin for deep infections, and carbapenems or ureidopenicillins with a $\beta$-lactamase inhibitor for more severe infections. The same guideline also considered the potential use of cotrimoxazole, vancomycin, linezolid or tigecycline if MRSA was suspected but did not mention any suspicion criteria. Although these guidelines are typically considered by HCPs, our study showed that the initial empirical antibiotic therapy covered the isolated pathogens of patients with clinically deteriorating ulcers in only $7.0 \%$ of the cases. Therapeutic failure was related to the presence of MDR organisms, namely MRSA.

In conclusion, our observational study provides a unique picture of the DFI pattern in our region. Both the prevalence and precocity of MDR organisms were alarmingly high and were probably related to indiscriminate antibiotic use. Fluoroquinolones, because of their pharmacological characteristics, safety and proven clinical effectiveness, are among the antimicrobial agents currently recommended by authoritative DFI guidelines. However, resistance has been directly linked to the use of these compounds, and the present study describes a statistical association that should encourage clinicians, and ultimately health authorities, to avoid their widespread use. By contrast, due to the high prevalence of MRSA in DFIs in our area, we suggest empirical anti-MRSA therapy followed by de-escalation to rationalize care and improve outcomes.

\section{Conflict of interest}

There are no conflicts of interest.

\section{Acknowledgments}

The authors would like to thank R. Oliveira and L. Prata from Associação Protectora dos Diabéticos de Portugal; J. Cabete, L. Mota-Capitão, C. Pereira-Alves, and L. Vasconcelos from Centro Hospitalar de Lisboa Central, EPE; F. Malheiro from Hospital de Curry Cabral.

\section{R E F E R E N C E S}

[1] Shaw JE, Sicree RA, Zimmet PZ. Global estimates of the prevalence of diabetes for 2010 and 2030. Diabetes Res Clin Pract 2010;87(1):4-14.

[2] Richard JL, Sotto A, Lavigne JP. New insights in diabetic foot infection. World J Diabetes 2011;2(2):24-32.

[3] Citron DM, Goldstein EJ, Merriam CV, Lipsky BA, Abramson MA. Bacteriology of moderate-to-severe diabetic foot 
infections and in vitro activity of antimicrobial agents. J Clin Microbiol 2007;45(9):2819-28.

[4] Tascini C, Piaggesi A, Tagliaferri E, Iacopi E, Fondelli S, Tedeschi A, et al. Microbiology at first visit of moderate-tosevere diabetic foot infection with antimicrobial activity and a survey of quinolone monotherapy. Diabetes Res Clin Pract 2011.

[5] Karchmer AW, Gibbons GW. Foot infections in diabetes: evaluation and management. Curr Clin Top Infect Dis 1994;14:1-22.

[6] National Institute for Health and Clinical Excellence. NICE clinical guideline 119: inpatient management of diabetic foot problems. London: National Institute for Health and Clinical Excellence; 2011.

[7] Joseph WS, Lipsky BA. Medical therapy of diabetic foot infections. J Am Podiatr Med Assoc 2010;100(5):395-400.

[8] Organization for economic co-operation and development (OECD). Health at a glance 2009: OECD indicators; 2009. Available from: www.oecd.org/dataoecd/32/34/ 43541373.pdf [last update September 2009, last accessed June 2011].

[9] Moet GJ, Jones RN, Biedenbach DJ, Stilwell MG, Fritsche TR. Contemporary causes of skin and soft tissue infections in North America, Latin America, and Europe: report from the SENTRY Antimicrobial Surveillance Program (1998-2004). Diagn Microbiol Infect Dis 2007;57(1):7-13.

[10] Schaper NC. Diabetic foot ulcer classification system for research purposes: a progress report on criteria for including patients in research studies. Diabetes Metab Res Rev 2004;20(Suppl. 1):S90-5.

[11] Executive summary: standards of medical care in diabetes2009. Diabetes Care 2009;32(Suppl. 1):S6-12.

[12] Lavery LA, Armstrong DG, Peters EJ, Lipsky BA. Probe-tobone test for diagnosing diabetic foot osteomyelitis: reliable or relic? Diabetes Care 2007;30(2):270-4.

[13] Alavi A, Niakosari F, Sibbald RG. When and how to perform a biopsy on a chronic wound. Adv Skin Wound Care 2010;23(3):132-40. quiz 141-2.

[14] Bonham PA. Swab cultures for diagnosing wound infections: a literature review and clinical guideline. J Wound Ostomy Continence Nurs 2009;36(4):389-95.

[15] Murray PR, Baron EJ, Jorgensen JH, Pfaller MA, Yolken RH. Manual of clinical microbiology. Washington, DC: ASM Press; 2003.

[16] Woo PC, Leung AS, Leung KW, Yuen KY. Identification of slide coagulase positive, tube coagulase negative Staphylococcus aureus by $16 \mathrm{~S}$ ribosomal RNA gene sequencing. Mol Pathol 2001;54(4):244-7.

[17] Clinical and Laboratory Standards Institute. Performance standards for antimicrobial disk susceptibility tests-M02A10 (includes M100-S20). Wayne, PA: Clinical and Laboratory Standards Institute; 2010.

[18] Bill TJ, Ratliff CR, Donovan AM, Knox LK, Morgan RF, Rodeheaver GT. Quantitative swab culture versus tissue biopsy: a comparison in chronic wounds. Ostomy Wound Manage 2001;47(1):34-7.

[19] Nelson EA, O'Meara S, Craig D, Iglesias C, Golder S, Dalton J, et al. A series of systematic reviews to inform a decision analysis for sampling and treating infected diabetic foot ulcers. Health Technol Assess 2006;10(12). iii-iv, ix-x, 1-221.

[20] Paterson DL, Bonomo RA. Extended-spectrum betalactamases: a clinical update. Clin Microbiol Rev 2005;18(4):657-86.

[21] Falagas ME, Koletsi PK, Bliziotis IA. The diversity of definitions of multidrug-resistant (MDR) and pandrug-resistant (PDR) Acinetobacter baumannii and Pseudomonas aeruginosa. J Med Microbiol 2006;55(Pt 12):1619-29.

[22] Prompers L, Schaper N, Apelqvist J, Edmonds M, Jude E, Mauricio D, et al. Prediction of outcome in individuals with diabetic foot ulcers: focus on the differences between individuals with and without peripheral arterial disease. The EURODIALE Study. Diabetologia 2008;51(5):747-55.

[23] Shakil S, Khan AU. Infected foot ulcers in male and female diabetic patients: a clinico-bioinformative study. Ann Clin Microbiol Antimicrob 2010;9:2.

[24] Zubair M, Malik A, Ahmad J. Clinico-microbiological study and antimicrobial drug resistance profile of diabetic foot infections in North India. Foot (Edinb) 2011;21(1):6-14.

[25] Bowler PG, Duerden BI, Armstrong DG. Wound microbiology and associated approaches to wound management. Clin Microbiol Rev 2001;14(2):244-69.

[26] Ng LS, Kwang LL, Yeow SC, Tan TY. Anaerobic culture of diabetic foot infections: organisms and antimicrobial susceptibilities. Ann Acad Med Singapore 2008;37(11):936-9.

[27] Dow G. Bacterial swabs and the chronic wound: when, how, and what do they mean? Ostomy Wound Manage 2003;49(5A Suppl.):8-13.

[28] Pellizzer G, Strazzabosco M, Presi S, Furlan F, Lora L, Benedetti $\mathrm{P}$, et al. Deep tissue biopsy vs. superficial swab culture monitoring in the microbiological assessment of limb-threatening diabetic foot infection. Diabet Med 2001;18(10):822-7.

[29] Slater RA, Lazarovitch T, Boldur I, Ramot Y, Buchs A, Weiss $\mathrm{M}$, et al. Swab cultures accurately identify bacterial pathogens in diabetic foot wounds not involving bone. Diabet Med 2004;21(7):705-9.

[30] Ferech M, Coenen S, Malhotra-Kumar S, Dvorakova K, Hendrickx E, Suetens C, et al. European Surveillance of Antimicrobial Consumption (ESAC): outpatient quinolone use in Europe. J Antimicrob Chemother 2006;58(2):423-7.

[31] Muller A, Mauny F, Talon D, Donnan PT, Harbarth S, Bertrand X. Effect of individual- and group-level antibiotic exposure on MRSA isolation: a multilevel analysis. J Antimicrob Chemother 2006;58(4):878-81.

[32] Direcção Geral da Saúde. Diabetic foot. National program for prevention and control of diabetes. In: Clinical Guidelines n`:05/PNPCD; 2010. 